\title{
Lactobacillus accelerates ISCs regeneration to protect the integrity of intestinal mucosa through activation of STAT3 signaling pathway induced by LPLs secretion of IL-22
}

\author{
Qihang Hou ${ }^{1} \cdot$ Lulu Ye $^{1} \cdot$ Haofei Liu' ${ }^{1}$ Lulu Huang ${ }^{1}$ Q Qian Yang ${ }^{1} \cdot \mathrm{JR}_{\text {Turner }}^{2,3,4} \cdot$ Qinghua Yu $^{1}$
}

Received: 7 June 2017 / Revised: 19 January 2018 / Accepted: 22 January 2018 / Published online: 19 February 2018

(c) ADMC Associazione Differenziamento e Morte Cellulare 2018

\section{Summary}

The regeneration of intestinal epithelial are maintained by continuous differentiation and proliferation of intestinal stem cells (ISCs) under physiological and pathological conditions. However, little is known about the regulatory effect of intestinal microbiota on its recovery ability to repair damaged mucosal barrier. In this study, we established intestinal organoids and lamina propria lymphocytes (LPLs) co-cultured system, plus mice experiments, to explore the protective effect of Lactobacillus reuteri $\mathrm{D} 8$ on integrity of intestinal mucosa. We found that only live L. reuteri D8 was effective in protecting the morphology of intestinal organoids and normal proliferation of epithelial stained with EdU under TNF- $\alpha$ treatment, which was also further verified in mice experiments. L. reuteri D8 colonized in the intestinal mucosa and ameliorated intestinal mucosa damage caused by DSS treatment, including improvement of body weight, colon length, pathological change, and proliferation level. The repair process stimulated by $L$. reuteri D8 was also accompanied with increased numbers of $\mathrm{Lgr}^{+}$and lysozyme ${ }^{+}$cells both in intestinal organoids and mice intestine. Furthermore, we demonstrated that D8 metabolite indole-3-aldehyde stimulated LPLs to secret IL-22 through aryl hydrocarbon receptor (AhR) and then induced phosphorylation of STAT3 to accelerate proliferation of intestinal epithelial, thus recovering damaged intestinal mucosa. Our findings indicate L. reuteri protects intestinal barrier and activates intestinal epithelial proliferation, which sheds light on treatment approaches for intestinal inflammation based on ISCs with probiotics Lactobacillus and daily probiotic consumption in heath foods.

Edited by D. Aberdam.

Electronic supplementary material The online version of this article (https://doi.org/10.1038/s41418-018-0070-2) contains supplementary material, which is available to authorized users.

Qinghua Yu

yuqinghua1981@njau.edu.cn

1 MOE Joint International Research Laboratory of Animal Health and Food Safety, College of Veterinary Medicine, Nanjing Agricultural University, Weigang 1, Nanjing, Jiangsu 210095, China

2 Department of Pathology, The University of Chicago, Chicago, IL, USA

3 Department of Pathology, Brigham and Women's Hospital, Boston, MA, USA

4 Department of Medicine,Division of Gastroenterology, Hepatology and Endoscopy, Brigham and Women's Hospital, Boston, MA, USA

\section{Introduction}

Integrity of the intestinal mucosa barrier is necessary to guarantee nutrients absorption, defend enteric pathogen invasion, and maintain mucosal immunity [1, 2]. The intestine is composed of a columnar epithelial mucosa with glandular invaginations called crypts. Intestinal stem cells (ISCs), located at the base of intestinal crypts, play a central role in governing proliferation and differentiation of the intestinal epithelium [3, 4]. Integrity of the intestinal mucosa is also important for recovery from inflammatory bowel disease (IBD) [5, 6]. Although the precise etiology of IBD remains unclear and controversial, the intestinal microbiota and integrity of mucosal epithelial function have been demonstrated to play key roles in its pathogenesis [79]. Although our understanding of the host pathways that regulate ISCs function is progressing, the effects of exogenous factors on ISCs biology remain poorly understood. Moreover, unlike other stem cells, ISCs co-exist with the 
Fig. 1 Building a co-cultured model with intestinal organoids and LPLs. a The co-culture model of LPLs and organoids. $\mathbf{b}$ Organoids cultured with LPLs were observed with a light microscope. Scale bar, $100 \mu \mathrm{m}$. c The growth status change of organoids from 1 to 6 days was observed with a light microscope. Scale bar, $50 \mu \mathrm{m}$
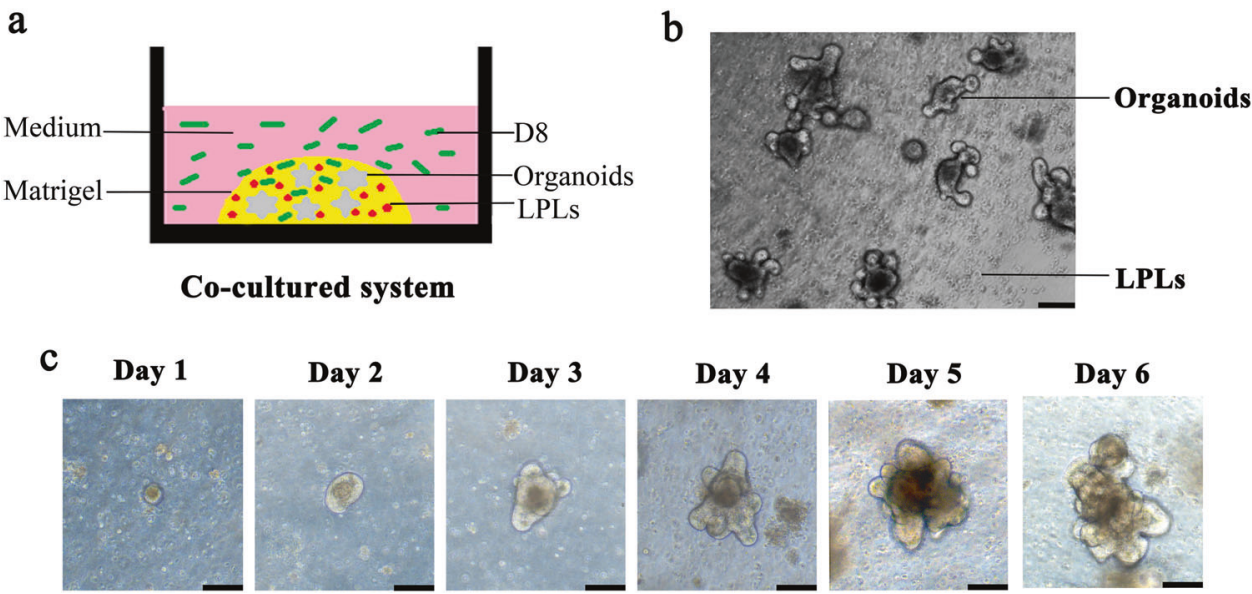

Day 2
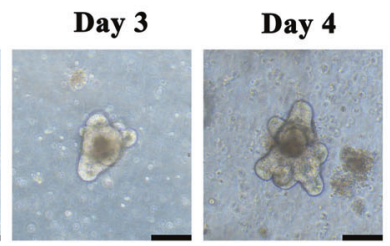

Day 5

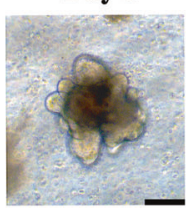

Day 6

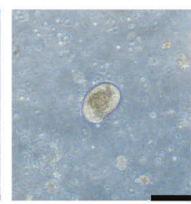

intestine to verify the protective effect of Lactobacillus on

intestinal microbiota, which may influence regeneration of the epithelium $[10,11]$.

Yogurt is consumed as a daily health food for its beneficial effects on regulating intestinal microbiota and modulating mucosal immunity [12-14]. Probiotic Lactobacillus strains are the main component of yogurt and are important components of intestinal microbiota; they produce antimicrobial agents and metabolic compounds that suppress the growth of other microorganisms and compete with other intestinal microbes for receptors and binding sites on intestinal mucosa $[15,16]$. The interactions between Lactobacillus and intestinal mucosa, including intestinal epithelial cells, have been studied for decades [17, 18]. Lactobacillus can also enhance the integrity of intestinal barrier and decrease disease phenotypes such as gastrointestinal infections and IBD [19-21]. However, the effects of Lactobacillus on ISCs remain unclear, which is attributed to the lack of a suitable in vitro model.

Lgr5 was recently identified as an ISCs marker that predominantly labels ISCs at the base of intestinal crypt [22]. Intestinal organoids containing all types of epithelial cells were first cultured in vitro and have been widely used for ISCs studies [23, 24]. Wang et al. [25] recently established culture conditions for a highly homogeneous population of ISCs. However, currently published models of intestinal organoid systems still lack enteric immune cells, which closely interact with ISCs in the crypt for communication [26]. Moreover, the mucosa immune and intestinal microbiota certainly play key roles in mucosal homeostasis and protecting against pathogens, infectious diseases, and inflammatory diseases [27, 28]. Group 3 innate lymphoid cells (ILC3s) can activate ISCs to regenerate via secretion of IL-22 and maintain intestinal epithelium [29].

The mechanism by which Lactobacillus in yogurt maintains the integrity of intestinal mucosa, especially its regulation of ISCs, remains unknown. In this study, we established a co-cultured system of mouse intestinal organoids with lamina propria lymphocytes (LPLs) from small intestinal epithelia. We hypothesized that Lactobacillus could stimulate the regeneration of ISCs to repair intestinal damage. The aim of this study was to explore the stimulatory effect of Lactobacillus reuteri D8 on LPLs and its effects on IL-22 secretion and ISCs regeneration, which could help further explain the protective effect of Lactobacillus on intestinal epithelia via the promotion of ISCs.

\section{Results}

\section{Building a co-cultured system with intestinal organoids and LPLs}

A co-cultured system consisting of intestinal organoids and LPLs was established in our study (Fig. 1a). Intestinal organoid were isolated from small intestine and cultured in Matrigel. LPLs were successfully isolated from small intestine and stained with Fixable Viability Dye efluor 660 to detect the viability with FACS. The survival ration of CD $45^{+}$LPLs maintained at a relative stable level within three days (Sup. Fig. 1a). Moreover, $\mathrm{CD} 45^{+} \mathrm{CD} 3^{+}$cells and $\mathrm{CD} 45^{+} \mathrm{CD}_{11 \mathrm{c}^{+}}$cells in LPLs represented about $49.3 \%$ and $2.83 \%$ respectively (Sup. Fig. 1b). ILC3s were identified as $\mathrm{CD}^{-}$cells that co-expressed CD45, CD127, and ROR $\gamma \mathrm{t}$ in previously reports [30, 31]. To define ILC3 cells in LPLs by flow cytometry, we first gated on $\mathrm{CD} 45^{+}$lineage-negative $\left(\mathrm{CD}^{-}\right)$lymphocytes and then examined ROR $\gamma \mathrm{t}^{+} \mathrm{CD} 127^{+}$ cells (ILC3 cells) in LPLs, which occupied $45.6 \%$ in $\mathrm{CD} 45^{+} \mathrm{CD}^{-}$region (Sup. Fig. 1b).

The co-cultured system was established with LPLs and intestinal organoids in Matrigel at a 7:1 ratio. Complete medium containing advanced DMEM/F12, 2 mM Glutamax, 10 mM HEPES, B27 supplement, N2 supplement, 50 $\mathrm{ng} / \mathrm{ml}$ mouse EGF, $100 \mathrm{ng} / \mathrm{ml}$ mouse Noggin, and $250 \mathrm{ng} /$ $\mathrm{ml} \mathrm{R}$-spondin 1 were added to the co-cultured system. The media were replaced every 2-3 days. We observed 

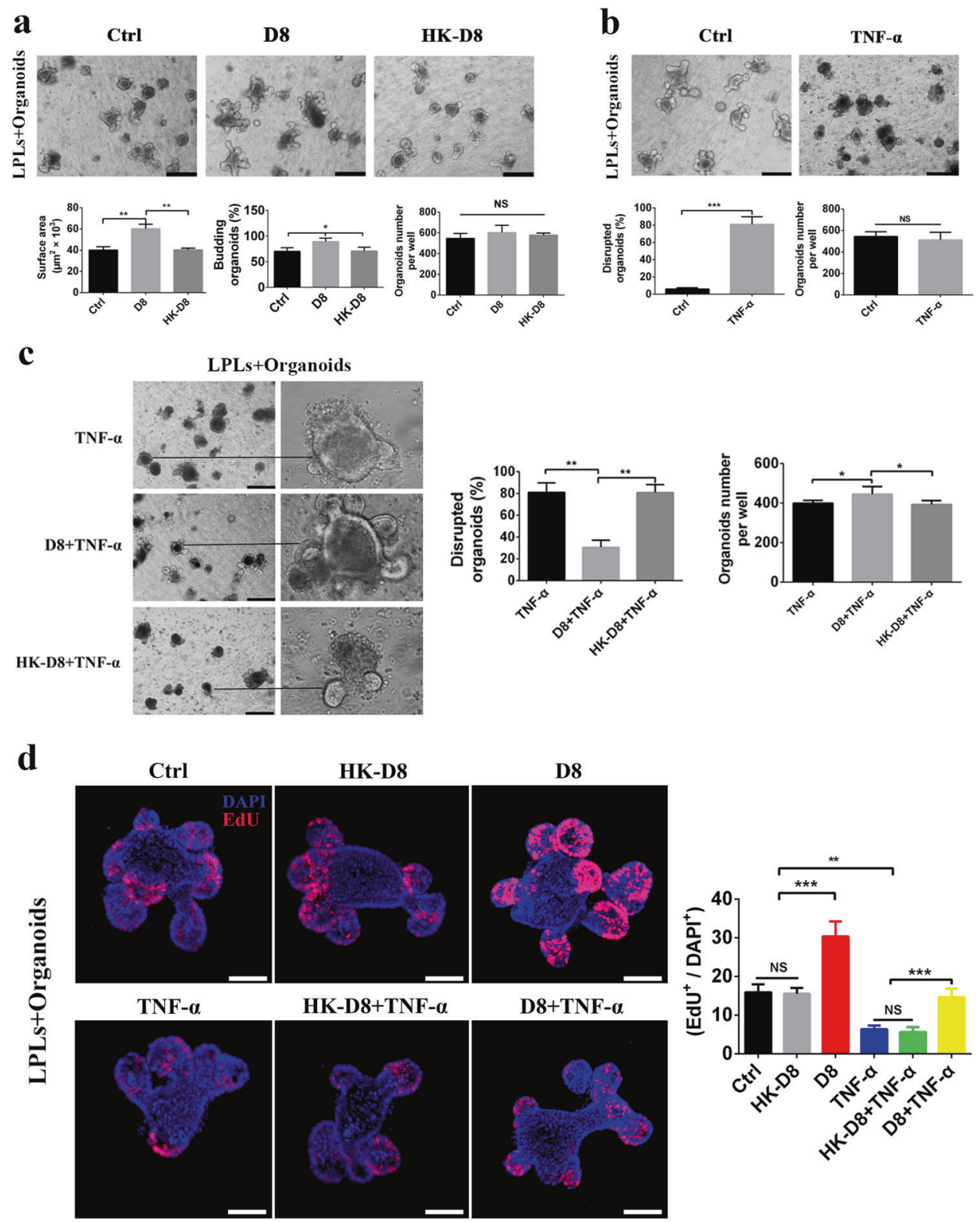

Fig. 2 D8 increases the growth of intestinal organoids and the recovery of intestinal organoids after damage caused by TNF- $\alpha$. a Size of organoids treated with/without D8 and HK-D8 $\left(1 \times 10^{4} \mathrm{CFU}\right.$ per well); $n=$ 50 organoids per group (day 3 ); total organoids number and budding organoids percentage of total organoids per well (day 3 ); $n=6$ wells per group. Scale bars, $200 \mu \mathrm{m}$. b Organoids were treated with TNF- $\alpha(60$ $\mathrm{ng} / \mathrm{ml}$ ) overnight, and the morphology of the organoids was observed with a light microscope. The number of total organoids and relative number of organoids with altered morphology per well were counted; $n$ $=50$ organoids per group. Scale bars, $200 \mu \mathrm{m}$. c Organoids were treated with TNF- $\alpha(60 \mathrm{ng} / \mathrm{ml})$ overnight with/without D8 and HK-D8 $\left(1 \times 10^{4}\right.$

intestinal organoids and LPLs growing in the wells (Fig. 1b). The organoids began to bud on the third day (Fig. 1c).

LPLs significantly increased the number of surface area (Sup. Fig. 1c) and 5-Ethynyl-2'-deoxyuridine (EdU)-
CFU per well). The number of total organoids and relative number of organoids with altered morphology per well were counted; $n=60$ organoids per group. Scale bars, $200 \mu \mathrm{m}$. d Organoids were stained with EdU (red). Nuclei are stained blue. EdU-positive cells were found in the transit-amplifying region with obvious differences in the percentages among the four samples; $n=30$ (control), $n=35$ (D8), $n=28$ (TNF- $\alpha$ ), $n=33$ (D8 + TNF- $\alpha$ ) organoids per group. Scale bar, $50 \mu \mathrm{m}$. Data are the mean $\pm \mathrm{SD}$; comparisons performed with $t$-tests (two groups) or analysis of variance (ANOVA) (multiple groups). $* P<0.05, * * P<$ $0.01, * * * P<0.001$. Data combined from at least three independent experiments unless otherwise stated

positive cells (Sup. Fig. 1d), as well as mRNA expression levels of ISC marker (Lgr5) and Paneth cell marker (Lyz1) compared to the organoids alone group (Sup. Fig. 1e). However, the number of organoids was not affected by the treatment of LPLs. 
a

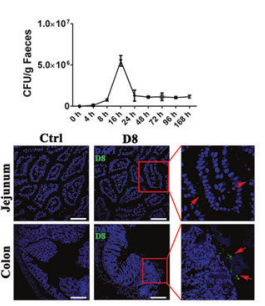

b

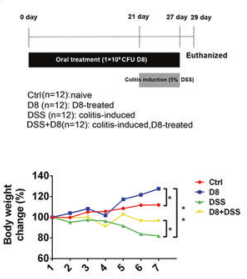

c

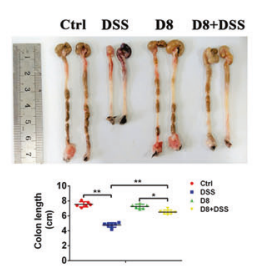

d
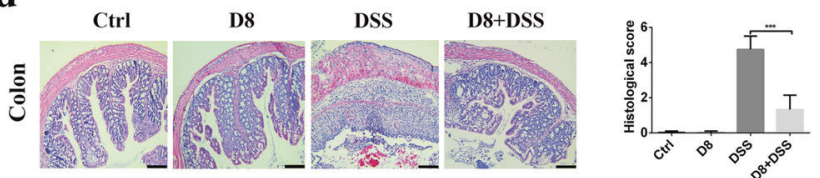

e
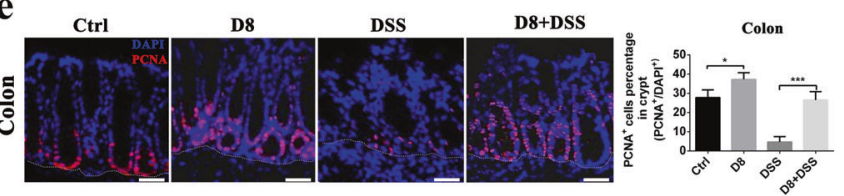

Fig. 3 D8 accelerates gut growth and ameliorates DSS-induced colitis in mice. a Mice were orally administrated with D8 $\left(10^{8} \mathrm{CFU}\right)$, the number of D8 in mice feces at indicated time points were detected by MRS plates containing tetracycline $(500 \mu \mathrm{g} / \mathrm{ml})$. D8 labelled with Dylight $488\left(10^{8} \mathrm{CFU}\right)$ was also administrated to mouse for $16 \mathrm{~h}$, the distribution of D8 in intestine was detected by confocal microscope. D8 (green), DAPI (blue). b Changes in body weight were monitored daily starting form 21 day and presented relative to the initial body weight; $n=12$ per group. c The colon lengths of mice treated with PBS, DSS, D8, or D8 + DSS. Treatment with DSS significantly reduced the colon length compared to the control group. D8 decreased the degree if reduction in colon length caused by DSS treatment. Scale bars, $200 \mu \mathrm{m}$. d Photomicrographs of the colons of mice treated with PBS, D8, DSS or DSS + D8. Note that treatment with D8 ameliorated DSS-induced colitis. Scale bars, $200 \mu \mathrm{m}$. e Confocal images (PCNA staining, red; and DAPI staining, blue) of colon of mice treated with PBS, D8, DSS or DSS + D8. The number of PCNA positive cells in

\section{D8 increases growth of intestinal organoids and recovery after TNF-a damage}

The co-cultured model containing intestinal organoids and LPLs was used to examine whether $L$. reuteri $\mathrm{D} 8$ or heat-killed D8 (HK-D8) could increase the growth of intestinal organoids or recovery of intestinal organoids after damage caused by TNF- $\alpha$. The intestinal organoids grew well after co-cultured with L. reuteri D8 or HK-D8 for $24 \mathrm{~h}$, exhibiting clear budding crypts. The surface areas and number of budding organoids co-cultured with L. reuteri $\mathrm{D} 8$ were significantly increased compared to the control and HK-D8 group (Fig. 2a). The number of disrupted organoids was significantly increased among organoids exposed to murine TNF- $\alpha(60 \mathrm{ng} / \mathrm{ml})$ (Fig. $2 b)$. Organoids will bud and differentiate into crypt and villi (Sup. Fig. 2a). However, damaged organoids became shrink and black, as well as buds lost (Sup. Fig. 2b). f
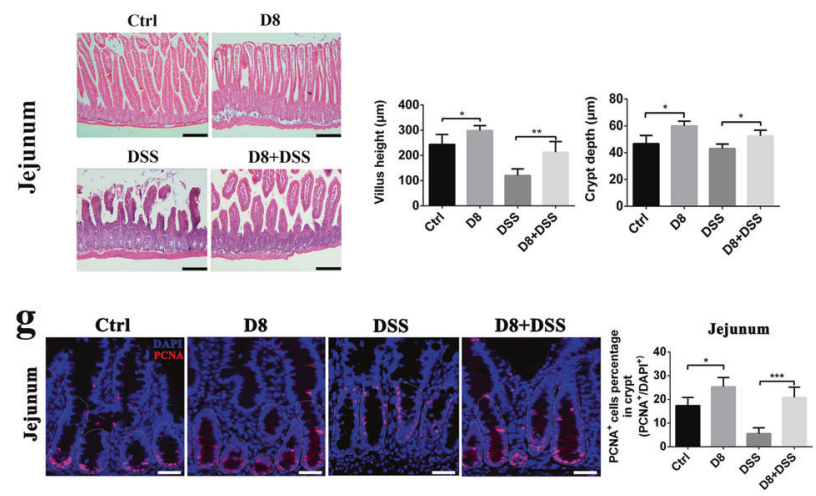

h

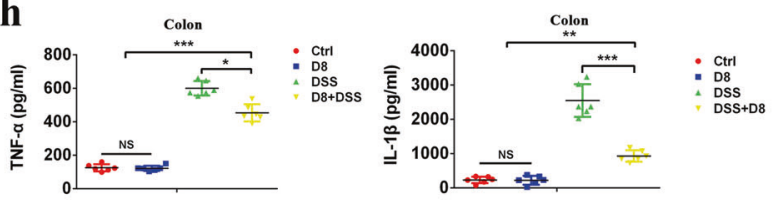

each crypt was detected. Scale bars, $200 \mu \mathrm{m}$ f Photomicrographs $(\times 200)$ of mice treated with PBS, D8, DSS or DSS + D8. Administration of D8 decreased DSS-induced intestinal morphometric changes in mice. Segments of the jejunum were processed to measure the height of the villus and the crypt depth. Scale bars, $200 \mu \mathrm{m}$. g Confocal images (PCNA staining, red; and DAPI staining, blue) of jejunum of mice treated with PBS, D8, DSS or DSS + D8. The number of PCNA positive cells in each crypt was detected. Scale bars, $200 \mu \mathrm{m}$. h Administration of D8 reversed the increased levels of TNF- $\alpha$ and IL$1 \beta$ in DSS-induced colitis. Note the increased levels of TNF- $\alpha$ and IL$1 \beta$ due to exposure to DSS. Treatment with D8 decreased the levels of TNF- $\alpha$ and IL- $1 \beta ; n=6$ per group. Data are the mean \pm SD. The comparisons were performed with $t$-tests (two groups) or analysis of variance (ANOVA) (multiple groups). $* P<0.05$, $* * P<0.01$, $* * * P<$ 0.001. Data combined from at least three independent experiments unless otherwise stated

However, L. reuteri D8 significantly ameliorated the damage to intestinal organoids caused by TNF- $\alpha$ (Fig. 2c). However, HK-D8 had no protective effect on intestinal organoids (Fig. 2c). The damage caused by TNF- $\alpha$, and the recovery induced by D 8 were time dependent (Sup. Fig. 2c).

The protective effects of $L$. reuteri D8 on intestinal organoids were also consistent with the proliferation status of the intestinal organoids after treatment with D8. TNF- $\alpha$ induced damage to the morphology of intestinal organoids with few EdU-positive cells in the crypt (Fig. 2d). However, D8 significantly increased the number of EdUpositive cells, thus explaining the enhanced surface area and number of budding organoids. Moreover, D8 also maintained more EdU-positive cells in the crypt damaged by TNF- $\alpha$, thus accelerating the recovery process. However, HK-D8 didn't show stimulatory effect on epithelial proliferation. 


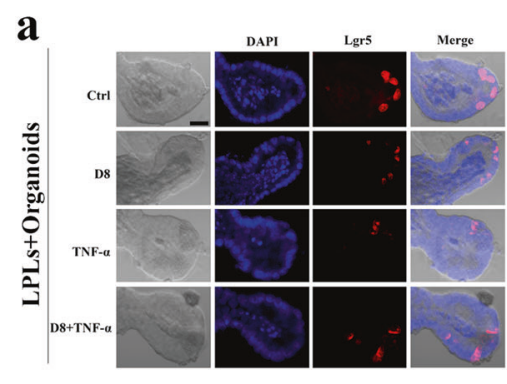

b
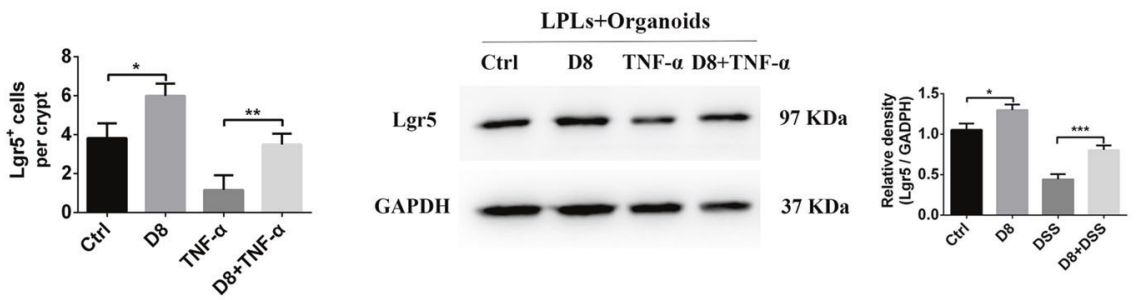

c

d
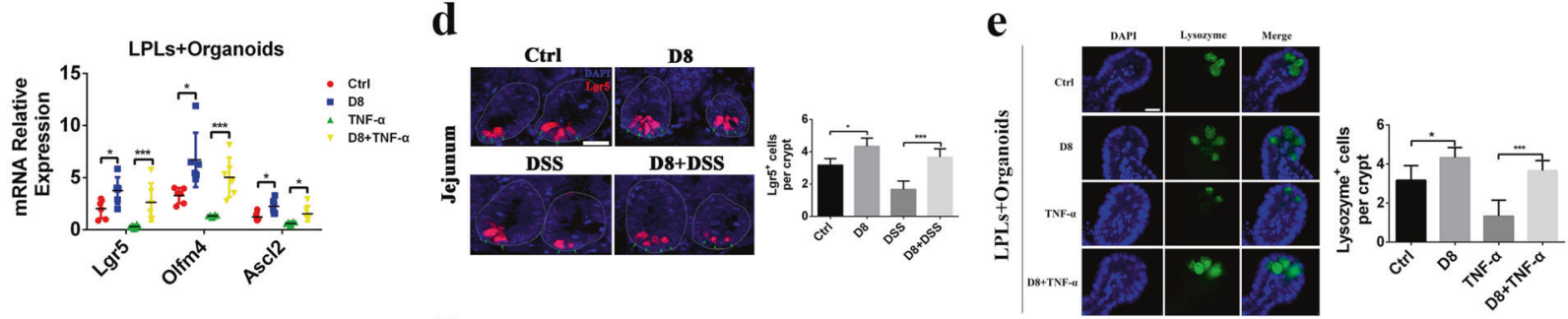

f

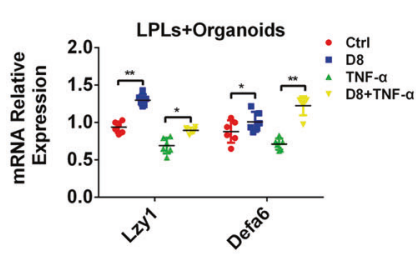

g

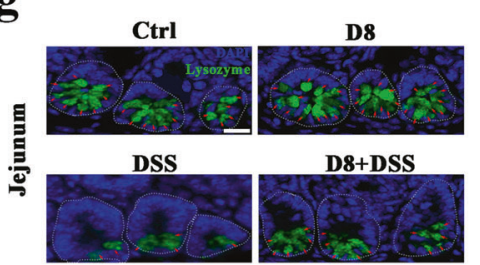

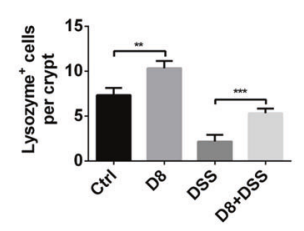

Fig. 4 D8 enhances the number of Paneth cells and stimulates ISCs regeneration. a Confocal images (Lgr5 staining, red; and DAPI staining, blue) of organoids cultured with/without D8 $\left(10^{4} \mathrm{CFU}\right.$ per well) and treated with/without TNF- $\alpha(60 \mathrm{ng} / \mathrm{ml})$ for $24 \mathrm{~h}$. The number of Lgr5-positive cells in each crypt were detected; $n=42$ (control), $n$ $=38$ (D8), $n=45(\mathrm{TNF}-\alpha), n=53$ (D8 $+\mathrm{TNF}-\alpha)$ organoids per group. Scale bar, $10 \mu \mathrm{m}$. b Western blot results of Lgr5 expression in organoids cultured with/without D8 $\left(10^{4} \mathrm{CFU}\right.$ per well $)$ and treated with/without TNF- $\alpha . n=3$ wells per group. $\mathbf{c}$ and $\mathbf{f}$ RT-qPCR was used to determine the relative mRNA expression of ISCs ( $L g r 5$, Ascl2, and Olfm 4 ) and Paneth cells (Lyz1 and Defa6) in organoids cultured with/without D8 and treated with/without TNF- $\alpha . n=6$ wells per group. d Confocal images (Lgr5 staining, red; and DAPI staining, blue) of the jejunum with different treatments. The number of Lgr5-

To further verify the effect of LPLs on the stimulation of organoid growth, organoids were cultured with D8 alone or in combination with LPLs. We found that L. reuteri D8 alone did not significantly increase the surface area of organoids (Sup. Fig. 3a, 3b).

\section{D8 accelerates gut growth and ameliorates dextran sulfate sodium (DSS)-induced colitis in mice}

After orally administrated with BacLight ${ }^{\mathrm{TM}}$ Green labelled L. reuteri $\mathrm{D} 8$, the colonization of fluorescence Lactobacillus was observed on the intestinal surfaces of jejunum and colon, which offered opportunities for D8 to contact with intestinal epithelium (Fig. 3a). Moreover, we collected the feces of the mice administrated with D8 and detected tetracycline-resistant Lactobacillus in MRS plate containing positive cells in each crypt were detected (circled by dotted line). Scale bar, $100 \mu \mathrm{m}$. e Confocal images (lysozyme staining, green; and DAPI staining, blue) of organoids cultured with/without D8 $\left(10^{4} \mathrm{CFU}\right.$ per well) and treated with/without TNF- $\alpha(60 \mathrm{ng} / \mathrm{ml})$. The number of lysozyme-positive cells in each crypt were detected; $n=50$ organoids per group. Scale bar, $10 \mu \mathrm{m}$. g Confocal images (lysozyme staining, green; and DAPI staining, blue) of the jejunum with different treatments. The number of lysozyme-positive cells in each crypt were detected (circled by dotted line). Scale bar, $100 \mu \mathrm{m}$. Data are the mean $\pm \mathrm{SD}$. The comparisons were performed with $t$-tests (two groups) or analysis of variance (ANOVA) (multiple groups). $* P<0.05, * * P<$ $0.01, * * * P<0.001$. Data combined from at least three independent experiments unless otherwise stated

tetracycline. We found Lactobacillus reached $10^{6} \mathrm{CFU} / \mathrm{g}$ in feces at $16 \mathrm{~h}$ and then reduced to a stable level during the experiment periods. Combined with the fluorescence results, it will be more convincible that $L$. reuteri $\mathrm{D} 8$ could colonize at the intestinal lumen and contact with the intestinal mucosa (Fig. 3a).

To confirm the effect of D8 on accelerating intestinal growth and ameliorating intestinal inflammation, C57BL/6 mice were orally administered D8. Non-treated and D8treated mice continuously gained weight, while DSS-treated mice began to lose weight after 3 days of DSS treatment. However, D8 greatly decelerated the weight loss (Fig. 3b). DSS treatment significantly reduced the colon length compared to the control group, while D8 minimized this reduction in colon length induced by DSS treatment (Fig. 3c). Histological examination revealed more extensive 
$\mathbf{a}$

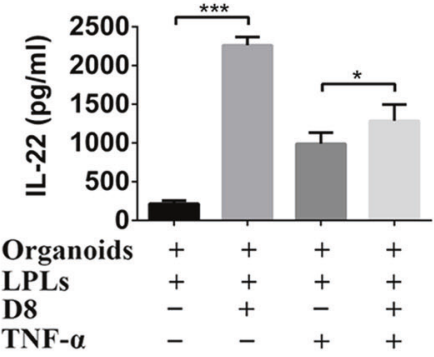

b

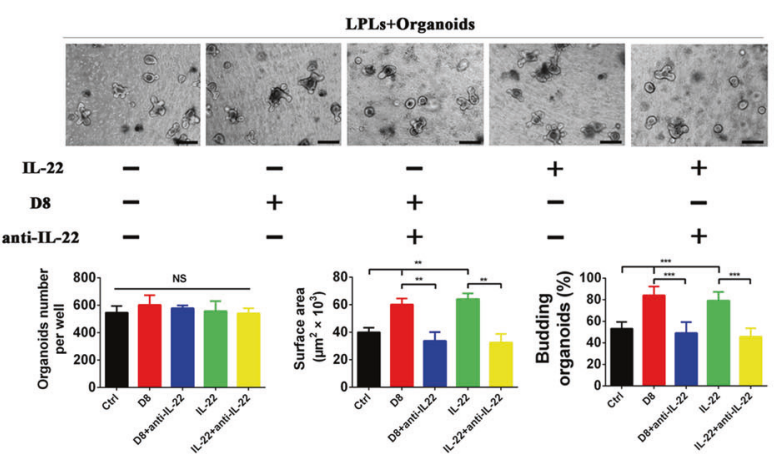

c
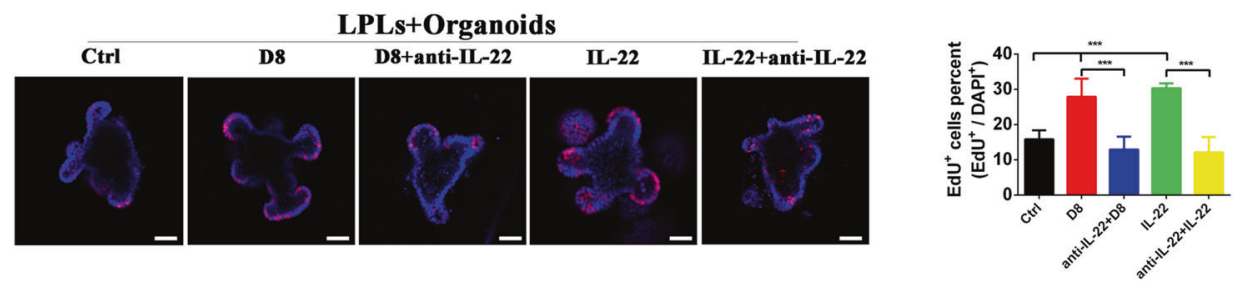

d

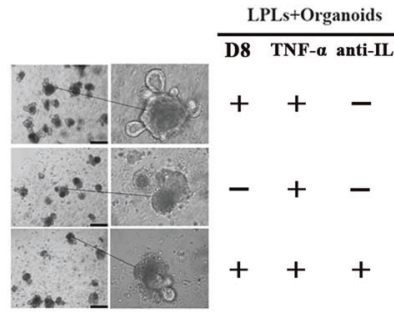

f

g

h
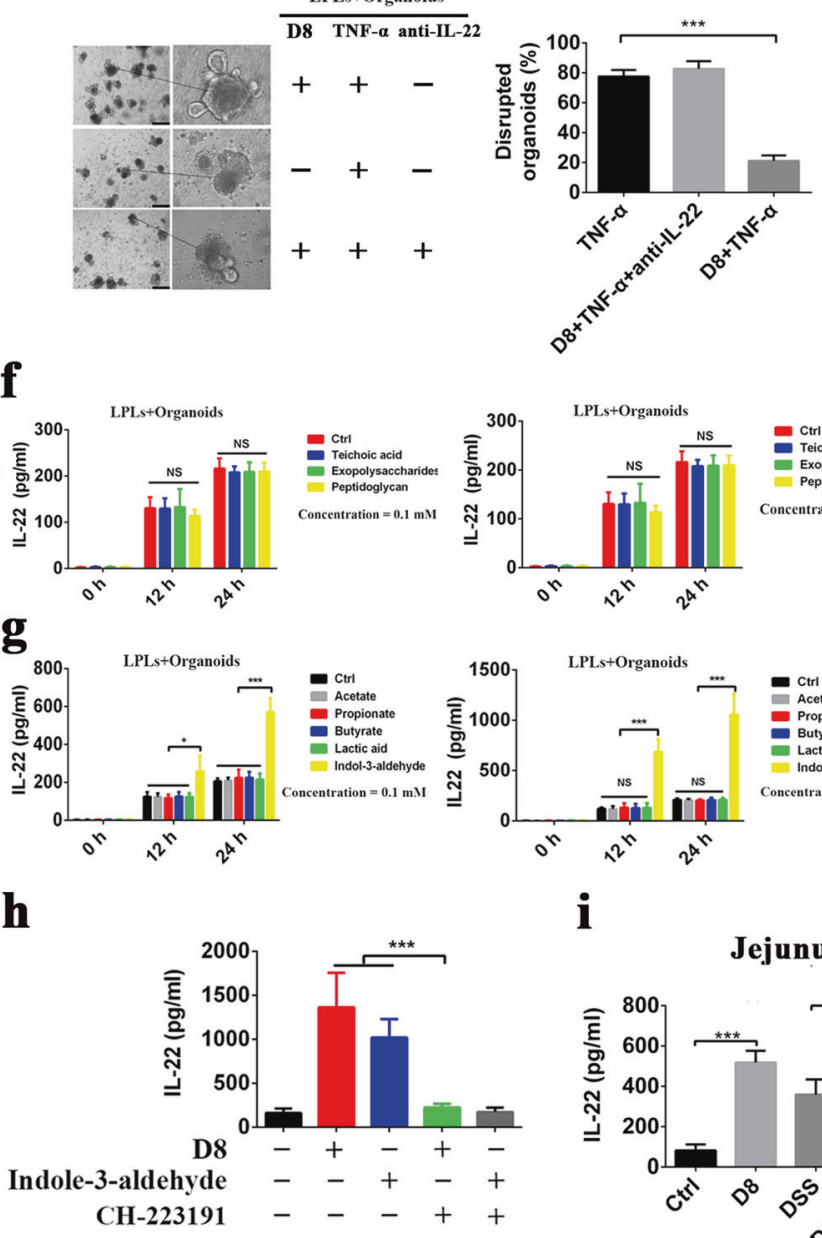

$$
\text { CH-223191 - }-\quad++
$$
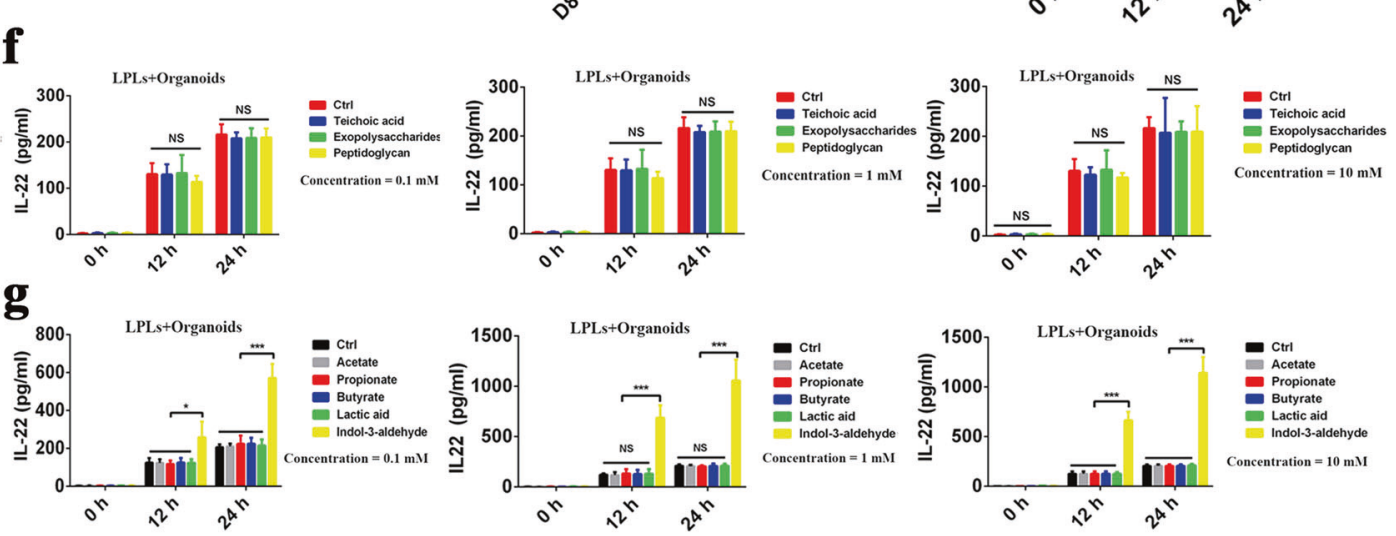

e

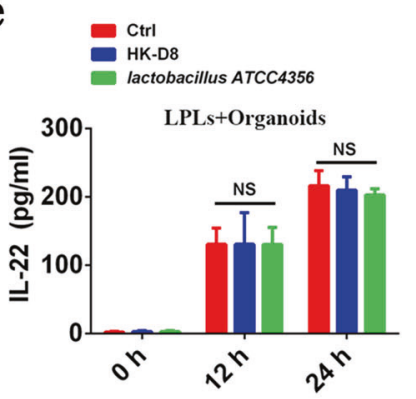

i

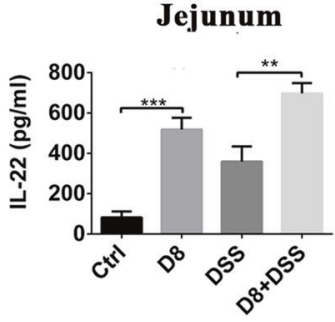

\section{Colon}

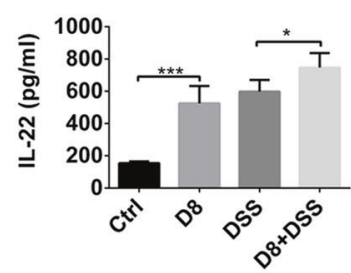


Fig. 5 D8 up-regulates IL-22 expression ex vivo and in vivo. a Organoids were treated with TNF- $\alpha(60 \mathrm{ng} / \mathrm{ml})$ or D8 $\left(1 \times 10^{4} \mathrm{CFU}\right.$ per well). The expression of IL-22 in the four groups was detected using an ELISA kit; $n=6$ well per group. b Size of organoids treated with/without D8 $\left(10^{4} \mathrm{CFU}\right.$ per well), IL-22 $(5 \mathrm{ng} / \mathrm{ml})$ or anti-IL-22 $(0.1 \mu \mathrm{g} / \mathrm{ml})$ for $24 \mathrm{~h} ; n=50$ organoids per group; organoids number and budding organoids percentage of total organoids per well; $n=6$ wells per group. Scale bars, $200 \mu \mathrm{m}$. c Organoids were stained with EdU (red). Nuclei were stained with DAPI (blue). EdU-positive cells were most distributed in the transit-amplifying region with obvious differences among the five groups; $n=30$ organoids per group. Scale bar, $50 \mu \mathrm{m}$. d After the organoids were treated, the morphologies of the organoids with different treatments were assessed by light microscopy. The relative number of organoids with altered morphology was counted; $n=50$ organoids per group. Scale bars, 200 $\mu \mathrm{m}$. e Organoids were treated with HK-D8 or Lactobacillus ATCC $4356\left(10^{4} \mathrm{CFU}\right.$ per well $)$ for $24 \mathrm{~h}$. The expressions of IL-22 were detected using an ELISA kit; $n=6$ well per group. f Organoids were treated with teichoic acid, exopolysaccharides, peptidoglycan $(0.1,1$, $10 \mathrm{mM}$ ) for $24 \mathrm{~h}$. The expression of IL-22 was detected using an ELISA kit; $n=6$ well per group. g, Organoids were treated with acetate, propionate, butyrate, lactic acid, and indol-3-aldehyde $(0.1$, $1,10 \mathrm{mM}$ ). The expression of IL-22 was detected using an ELISA kit; $n=6$ well per group. $\mathbf{h}$ Organoids were cultured with/without D8 $\left(1 \times 10^{4} \mathrm{CFU}\right.$ per well $)$ or indole-3-aldehyde $(1 \mathrm{mM})$ and treated with/without AHR inhibitor CH-223191 (1 mM). i Mice were treated with PBS, D8, DSS or DSS + D8. The expression of IL-22 in the four different groups was detected using an ELISA kit; $n=6$ mice per group. Data are the mean $\pm \mathrm{SD}$; comparisons performed with $t$ tests (two groups) or analysis of variance (ANOVA) (multiple groups). $* P<0.05, * * P<0.01, * * * P<0.001$. At least three independent experiments were performed

bowel edema and dense infiltration of the superficial layers of the mucosa in colonic tissues, as well as pathological bleeding in the colonic lumen and muscular layer in the DSS group (Fig. 3d). The pathological changes caused by DSS were ameliorated in the D8 group, which exhibited generally normal colonic structure and no bleeding. Similar pathological damage was also detected in the jejunum, which had a reduced villus height. D8-treated mice showed longer villus and greater depth than untreated mice. Moreover, D8 ameliorated the intestinal structure damage, with markedly increased villus height and crypt depth compared to the DSS group (Fig. 3f).

The protective effect of D8 on intestine was also consistent with the proliferation status of the intestinal epithelial after treatment with D8. DSS-induced damage to the morphology of jejunum and colon with few proliferating cell nuclear antigen (PCNA)-positive cells. However, D8 not only significantly stimulated the proliferation of intestinal epithelial with PCNA staining under physiological status, but also improved the proliferation status reduced by DSS treatment to accelerate the recovery process (Fig. 3e,g).

The reduced colon length and pathological changes were consistent with the increased protein expression of proinflammatory cytokines IL- $1 \beta$ and TNF- $\alpha$. The protein expression levels of TNF- $\alpha$ and IL- $1 \beta$ in mice treated with both DSS and D8 were significantly reduced compared with the DSS-treated group (Fig. 3h).

\section{D8 increases the number of Paneth cells and stimulates ISCs regeneration after damage}

ISCs are critical for damage-induced intestinal regeneration. In this study, we evaluated the number of red fluorescencelabeled Lgr5-positive cells in the crypt. We observed a higher percentage of Lgr5-positive cells in D8-treated intestinal organoids cultured with LPLs compared to untreated intestinal organoids cultured with LPLs alone. Upon being damaged by TNF- $\alpha$, D8 significantly increased the number of Lgr5-positive cells compared to the TNF- $\alpha$ group (Fig. 4a). The stimulatory effect of D8 on ISCs was further verified by the protein expression of Lgr5 (Fig. 4b) and mRNA expression of ISC markers ( $\operatorname{gr} 5$, Olfm4, and Ascl2) (Fig. 4c). The stimulatory effect of D8 on Lgr5positive cells in intestinal organoids was further verified in mice. D8 increased the number of Lgr5-postive cells and ameliorated the decrease caused by DSS in jejunum (Fig. 4d).

Paneth cells could modulate the proliferation and differentiation of ISCs through secretion of EGF, delta-like 1/ 4, and Wnt-3 [32]. In this study, we found that D8 significantly increased the number of lysozyme-positive cells compared to the control group both in organoids and jejunum (Fig. 4e,g). However, TNF- $\alpha$ treatment significantly reduced the number of lysozyme-positive cells compared to the control group and mRNA expression levels of Paneth cell markers (Lyzl and Defa6) (Fig. 4f). D8 ameliorated the decrease of lysozyme-positive cells caused by TNF- $\alpha$ in organoids or DSS in mice (Fig. 4e,g).

\section{D8 up-regulates IL-22 expression ex vivo and in vivo}

IL-22 promoted ISC-mediated epithelial regeneration [29]. In this study, when co-cultured with LPLs, Lactobacillus D8 could significantly stimulate the secretion of IL-22 compared to control group, which could explain the protective effect of Lactobacillus on intestinal epithelia (Fig. 5a). Without LPLs in the cultural model, L. reuteri D8 alone could not induce the secretion of IL-22 (Sup. Fig. 3c). However, D8 alone could induce the LPLs to secret IL-22 without organoids (Sup. Fig. 3d). Interestingly, TNF- $\alpha$ treatment also increased IL-22 production, which may be attributed to the initiation of the self-repair system under intestinal damage. However, D8 further enhanced the efficiency of repair system by increasing IL-22 production (Fig. 5a). Moreover, the growth stimulation effects of D8 and IL-22 on intestinal organoids, including surface area, budding, and proliferation, could be inhibited by addition of 
a

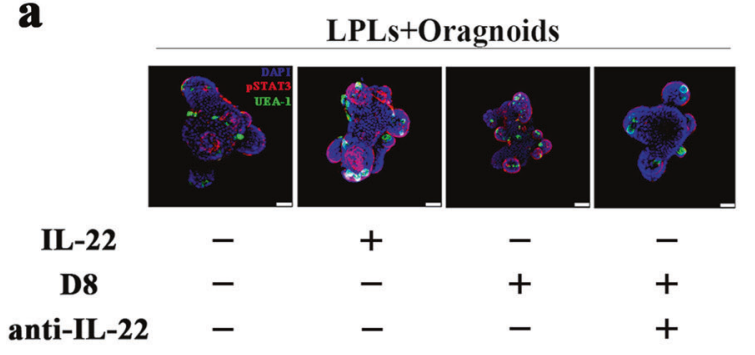

b

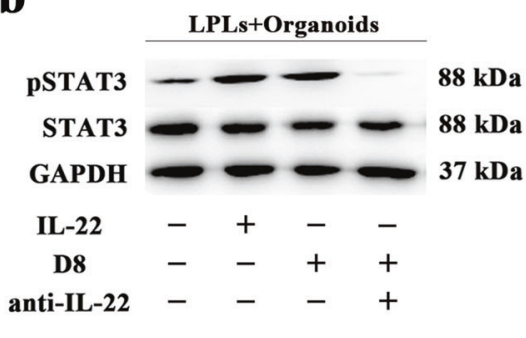

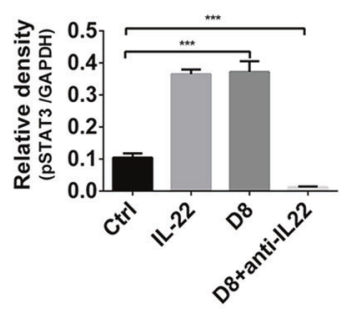

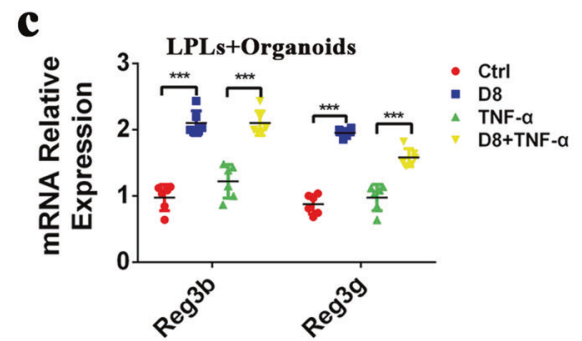

d

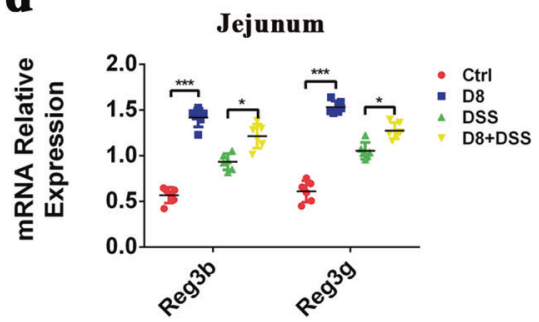

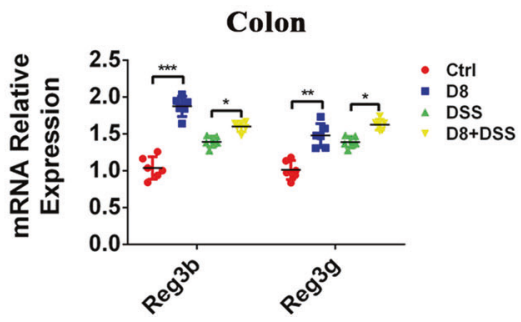

Fig. 6 D8 activates pSTAT3 and the Reg3 signal pathway. a Confocal images of organoids of different treatments (pSTAT3 staining, red; UAE-1 staining, green; DAPI staining, blue). Obvious differences in the percentages of PSTAT3-positive and UAE-1-positive cells were observed among the four samples; $n=20$ organoids per group. Scale bar, $50 \mu \mathrm{m}$. b Western blot results of pSTAT3 in organoids treated with IL-22 $(5 \mathrm{ng} / \mathrm{ml})$ or D8 $\left(1 \times 10^{4} \mathrm{CFU}\right.$ per well $)$ with/without antiIL-22 $(0.1 \mu \mathrm{g} / \mathrm{ml}) ; n=3$ wells per group. c The relative mRNA

anti-IL-22 (Fig. 5b,c). The protection effect of D8 on the morphology of intestinal organoids damaged by TNF- $\alpha$ was also inhibited by the addition of anti-IL-22 (Fig. 5d). Combined with above results, we found that D8 stimulated LPLs to secret IL-22 and proliferation of intestinal epithelial, thus facilitating efficient recovery under TNF- $\alpha$ treatment.

However, HK-D8 and L. acidophilus ATCC 4356, as well as several microbe associated molecular patterns (MAMPs), such as peptidoglycan, teichoic acids, polysaccharides, at different concentrations $(0.1,1,10 \mathrm{mM})$, could not significantly up-regulate IL-22 expression in the co-cultured model (Fig. 5e,f). Similar results were also detected for metabolites of gut microbiota such as acetate, propionate, butyrate, and lactic acid at different concentrations $(0.1,1,10 \mathrm{mM})$. Whereas indol-3-aldehyde, the tryptophan metabolism by $L$. reuteri, that contributes to activate AhR, up-regulated IL-22 expression at different concentrations $(0.1,1,10 \mathrm{mM})$ in the co-cultured model (Fig. 5g). Furthermore, the IL-22 induction effect of D8 or indole-3-aldehyde was inhibited by the addition of AhR inhibitor CH-223191 (Fig. 5h). Considering the indole-3aldehyde is the metabolite of $L$. reuteri $\mathrm{D} 8$, which further confirmed D8 activated AhR to induce the induction of IL22. The stimulatory effect of D8 on IL-22 secretion was also verified in vivo. D8 increased IL-22 production both in jejunum and colon compared to control group. Furthermore, expressions of Reg3b and Reg3g in organoids treated with D8 $(1 \times$ $10^{4} \mathrm{CFU}$ per well) or TNF- $\alpha(60 \mathrm{ng} / \mathrm{ml})$ for $24 \mathrm{~h} ; n=6$ wells per group. d The relative mRNA expression of Reg3b and Reg3g in the jejunum and the colon treated with/without D8 and with/without DSS, $n=6$ mice per group. Data are the mean $\pm \mathrm{SD}$. The comparisons were performed with $t$-tests or analysis of variance (ANOVA). $* P<0.05$, $* * P<0.01, * * * P<0.001$. At least three independent experiments were performed

D8 enhanced IL-22 production under DSS treatment (Fig. 5i).

\section{D8 activates the PSTAT3 signaling pathway through the secretion of IL-22}

The secretion of IL-22 by LPLs stimulated with D8 to protect the intestinal epithelial barrier was also verified by treating organoids with anti-IL-22. D8 treatment stimulated the budding of intestinal organoids with increased pSTAT3positive cells, which were shown in red fluorescence. A similar phenomenon was observed upon the addition of IL22 to the culture medium. However, the addition of anti-IL22 inhibited the stimulatory effect of D8 on organoids (Fig. 6a). The immunofluorescence results were further verified by Western blot (Fig. 6b), which showed that D8 stimulated the secretion of IL-22 to drive phosphorylation of STAT3 in intestinal organoids.

RT-qPCR was performed to detect the expression of Reg3b and Reg3g in the organoids. As expected, D8 treatment increased the relative mRNA expression of Reg $3 b$ and Reg3g compared with control group. Moreover, D8 enhanced Reg3b and Reg3g expression under pathological status compared to TNF- $\alpha$-induced damage to organoids (Fig. 6c). The increased expressions of Reg $3 b$ and Reg3g was also verified in the jejunum and colon of mice (Fig. 6d). 


\section{Discussion}

Probiotics are live microbial food supplements or components of bacteria that have been shown to exert beneficial effects on human health [33-35]. Lactobacillus has been widely used in clinical studies and in dairy foods for the prevention and treatment of various gastrointestinal infections and inflammatory conditions, as it protects the integrity of intestinal mucosa [36, 37]. According to previous papers, $L$. reuteri has the ability to active the AhR and induce LPLs to secret IL-22 [38, 39]. Intestinal epithelial proliferation and replacement are critical for maintaining the intestinal mucosa structure [40]. However, little is known regarding the effect of Lactobacillus on ISCs. Previous published studies have also demonstrated that ILC3 of LPLs could produce IL-22 [2, 29]. In this study, we first established the intestinal organoids and LPLs co-cultured model, and found that LPLs secreted IL-22 at low level to stimulate the organoids growth and epithelial proliferation. We further demonstrated that $L$. reuteri D8 stimulated the growth of intestinal organoids, with increased surface areas and organoid budding, and protected organoid morphology under TNF- $\alpha$ treatment. This protective effect of D8 against TNF- $\alpha$ is consistent with the increased proliferation of cells in organoids observed via EdU staining. However, HK-D8 could not protect the normal morphology and proliferation of organoids from TNF- $\alpha$ damage. This is the first study to explain the protective effect of Lactobacillus on intestinal epithelia by facilitating the proliferation of ISCs.

The epithelial barrier limits interactions between luminal contents such as the intestinal microbiota, preventing intestinal inflammation [41]. A previous study demonstrated that, after treatment with DSS, the proinflammatory cytokine TNF- $\alpha$ in the intestine was significantly increased [42]. We orally administered mice with DSS to verify the protective effect of D8 in intestinal organoids treated with TNF- $\alpha$. DSS treatment significantly increased TNF- $\alpha$ and IL-1 $\beta$, which was ameliorated by D8 in this study. The reduced body weight and colon length were also mitigated by $\mathrm{D} 8$, as well as the pathological changes in the jejunum and colon of mice. The transit-amplifying cells located in the crypt are the main impetus to replacing damaged epithelia. Furthermore, D8 increased the crypt depth in physiological conditions and pathological status under DSS treatment, indicating the stimulatory effect of D8 on progenitor cells. This phenomenon is consistent with the increased proliferation of intestinal epithelial in jejunum and colon stained with PCNA. Moreover, the pathological improvement could also be explained by the control of TNF- $\alpha$ and IL- $1 \beta$ secretion.

$\mathrm{Lgr5}^{+}$(a marker for ISCs) ISCs can generate all epithelial cell types of mature intestinal epithelium [22, 23, 43]. Paneth cells protect the epithelial barrier with antimicrobial molecule secretion and maintain the epithelium by providing an epithelial niche for ISCs [32, 44]. Here, we found that D8 increased the protein expression of Lgr5 and the number of $\mathrm{Lgr}^{+}$positive cells in the intestinal organoids under TNF- $\alpha$ damage, which was also further verified by the increased mRNA expression of ISCs markers (Lgr5, Olfm4, and Ascl2). This phenomenon further confirmed our hypothesis that Lactobacillus can stimulate the proliferation of epithelial to protect the integrity of the intestinal mucosa. The increased proliferation of ISCs by D8 was also supported by the enhanced number of Paneth cells, which can secret EGF, TGF- $\alpha$, Wnt3, and the Notch ligand D114 for the maintenance of ISCs [32]. Moreover, higher mRNA expression levels of Lyz1 and Defa6 were also detected. The capacity of stem cells to survive is linked to their ability to repair damage and induce proliferation in tissue.

Previous reports have demonstrated that IL-22 maintained gut epithelial integrity [45, 46]. A recent study showed that IL-22 was important for augmenting ISCmediated epithelial regeneration and epithelial proliferation $[29,47]$. In this study, we demonstrated that D8 increased the secretion of IL-22 under TNF- $\alpha$ treatment to accelerate ISC-mediated epithelial regeneration. The induction of IL22 by $L$. reuteri $\mathrm{D} 8$ was attributed to the activation of $\mathrm{AhR}$, which was further confirmed by the treatment with indol-3aldehyde and AhR inhibitor. However, HK-D8, L. acidophilus ATCC 4356, several MAMPs (peptidoglycan, teichoic acids, polysaccharides) and microbiota metabolites (acetate, propionate, butyrate and lactic acid) could not significantly stimulate the secretion of IL-22. These results indicated that the protective effect of intestinal epithelial by Lactobacillus is strain specificity. We also found that IL-22 was increased in mice administrated with D8 in vivo. The combination of increased $\mathrm{Lgr}^{+}$cells and Paneth cells, and the accelerated proliferation ratio could explain the stimulatory effect of D8 on ISCs to protect epithelial. This explanation was further confirmed by the addition of antiIL-22, which resulted in an enhanced number of disrupted organoids.

We also found that D8 stimulated phosphorylation of the STAT3 signaling pathway, which could be inhibited by the addition of anti-IL-22. STAT3 activation by IL-22 is consistent with the result of previous report [29]. Moreover, IL22-mediated STAT3 activation could further stimulate proliferation of the crypt. Increased expression of antimicrobial peptides (AMPs) Reg3 $\beta$ and Reg3 $\gamma$ were also detected, which could restrict bacterial colonization of mucosal surfaces and reduce bacterial translocation to protect mucosa [48].

In summary, this is the first report to identify a mechanism by which Lactobacillus increases intestinal epithelial proliferation and reduces the intestinal damage 

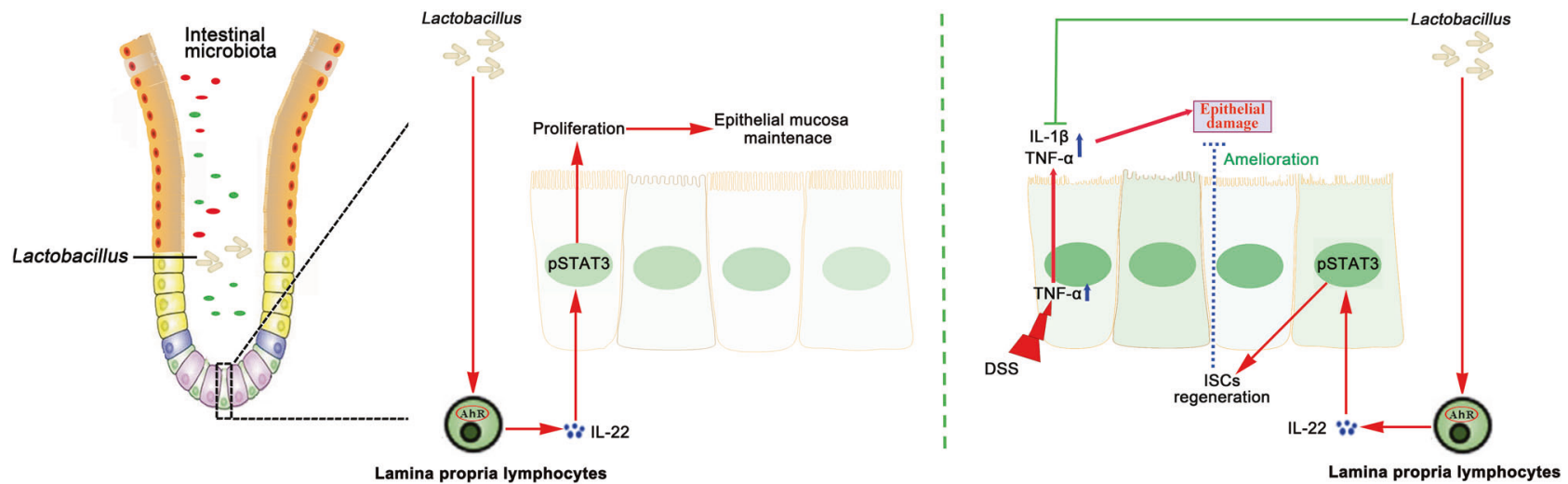

Fig. 7 Our model depicts the protection conferred by Lactobacillus on the intestinal epithelial barrier via the modulation of ISCs. Lactobacillus stimulated LPLs to secret IL-22 via AhR and then activated phosphorylation of STAT3 to accelerate ISCs regeneration and thus maintain the epithelial barrier. Some factors, such as DSS, stimulated

the intestinal mucosa to secret IL- $1 \beta$ and TNF- $\alpha$, and then induced epithelial damage and intestinal inflammation. However, Lactobacillus stimulated ISCs proliferation through the pSTAT3 pathway activated by IL-22 and inhibited inflammatory cytokine secretion to protect the mucosa barrier

caused by TNF- $\alpha$ treatment. Moreover, we demonstrate that D8 stimulates LPLs to secret IL-22 through AhR and then activates phosphorylation of STAT3 to accelerate ISCs regeneration and, thus, recovery of intestinal epithelial structure under TNF- $\alpha$ treatment (Fig. 7).

\section{Materials and methods}

\section{Animals and bacteria strains}

C57BL/6 mice (4 weeks old, specific-pathogen-free [SPF]) were purchased from the Animal Research Centre of Yangzhou University. All the animal studies were approved by the Institutional Animal Care and Use Committee (IACUC) of Nanjing Agricultural University, and the National Institutes of Health guidelines for the performance of animal experiments were followed. The $L$. reuteri D8 strain was isolated from pig intestinal lumen and further confirmed as $L$. reuteri strain through 16s RNA sequencing (GenBank: MF850249), which are tetracycline resistance. Lactobacillus acidophilus ATCC 4356 was purchased from the China Committee for Culture Collection of Microorganisms. All Lactobacillus strains were grown in MRS ager medium at $37^{\circ} \mathrm{C}$.

\section{Crypt isolation and intestinal organoid culture}

Intestinal organoids were obtained from intestines of 4week-old C57BL/6 mice, as previously described [23, 24]. Briefly, pieces of intestine were dissected and washed with phosphate-buffered saline (PBS) and then chopped into pieces. After thorough washing in PBS, the pieces were incubated in $2 \mathrm{mM}$ EDTA for $30 \mathrm{~min}$ at $4{ }^{\circ} \mathrm{C}$ on a rocking platform. The mixture was passed through a $70 \mu \mathrm{m}$ cell

strainer, and crypt fractions were isolated and purified through centrifugation. Then the crypt fractions, mixed with $50 \mu \mathrm{l}$ of Matrigel (BD Bioscience/Corning), were plated in 24-well plates in Advanced DMEM/F12 supplemented with penicillin-streptomycin, $10 \mathrm{mM}$ HEPES, $2 \mathrm{mM}$ glutamine, N2, B27 (all from Gibco, Life Technologies), and EGF (50 $\mathrm{ng} / \mathrm{ml}$, Peprotech) and Noggin (100 ng/ml, Peprotech).

The detailed methods of organoids passage and counting are listed as follows. Firstly, place tissue culture plate on ice to thaw Matrigel and then aspirate media, and add $1 \mathrm{ml}$ of cold PBS to each well. Pipette up and down until no solid Matrigel chunks remain. Spin down for $4 \mathrm{~min}$ at $900 \mathrm{rpm}$ at $4{ }^{\circ} \mathrm{C}$ and remove PBS. Resuspend in $50 \mu \mathrm{l} \mathrm{Matrigel} / \mathrm{well}$ and allow Matrigel to polymerize, then overlay each well with $650 \mu \mathrm{l}$ culture medium. The number of organoids were counted with cell counting chamber under light microscopy.

\section{Establishing co-cultured system containing LPLs and intestinal organoids}

For co-cultured experiments, LPLs were isolated from lamina propria of the small intestine. First, small intestine were cut lengthwise and washed with PBS, and incubated in EDTA/IEL solution (5\% FBS, 1\% penicillin/streptomycin, $10 \mathrm{mM}$ HEPES buffer, $1 \%$ L-glutamine, $1 \mathrm{mM}$ EDTA, all from Gibco, Life Technologies) for $30 \mathrm{~min}$. Then shake the small intestine samples for 2 min by hand and discard the supernatant. The intestine were then treated with collagenase solution (RPMI1640, $1 \%$ glutamine, $10 \mathrm{mM}$ HEPES, $1 \%$ penicillin/streptomycin, 5\% FBS, $1 \mathrm{mg} / \mathrm{ml}$ collagenase D and $1 \mathrm{U} / \mathrm{ml}$ DNase I, all from Gibco, Life Technologies) incubated for $30 \mathrm{~min}$ in a $37^{\circ} \mathrm{C}$ shaker. Afterwards, the supernatant was filtered with $40 \mu \mathrm{m}$ cell strainer. Afterwards, the filtrate were centrifuged at 2000 rpm for $5 \mathrm{~min}$ and washed with RPMI solution without 
enzymes. Then the cell suspension was centrifuged and discarded the supernatant. The cell was resuspended with $40 \%$ Percoll solution (in PBS) and was overlaid with an $80 \%$ Percoll solution. After spinning, the interface containing the LPLs were aspirated and washed in medium.

LPLs were stained with Fixable Viability Dye efluor 660 (1:100, 65-0864-14, eBioscience) or fluorescent mAbs specific for mouse FITC-CD45 (1:100, 110452, eBioscience), APC-CD3 (1:100, 17A2, BD Biosciences), APC-CD11c (1:100, N418, BD Biosciences), PerCP-cy5.5CD127 (1:100, 45-1278-42, eBioscience) and at $4{ }^{\circ} \mathrm{C}$ for 30 min. After washing three times with PBS, the cells were phenotypically analyzed by FACS (BD FACSCalibur). For nuclear staining, Foxp3/transcription factor staining buffer (1:100, A24261, eBioscience) set was used in combination with PE-ROR $\gamma t$ (1:100, 12-6981, eBioscience).

Then the isolated LPLs were cultured with intestinal organoids at ratio of 7:1 in Matrigel. L. reuteri D8, HK-D8, L. acidophilus ATCC $4356\left(10^{4} \mathrm{CFU} /\right.$ well $)$ and murine TNF- $\alpha$ ( $60 \mathrm{ng} / \mathrm{ml}$, Peprotech), acetate, propionate, butyrate, lactic acid, indlo-3-aldehyde, teichoic acid, expolysacchairdes, peptidoglycan $(0.1,1,10 \mathrm{mM}$, Sigma) were added independently or simultaneously to the culture medium for $24 \mathrm{~h}$. Murine IL-22 (5 ng/ml, Peprotech), antiIL-22 neutralizing antibody $(0.1 \mu \mathrm{g} / \mathrm{ml}$, AF582, RD) and AhR inhibitor CH-223191 (1 mM, C8124, Sigma) were also used to treat organoids for $24 \mathrm{~h}$.

\section{Intestinal organoids measurement}

If all organoids in a well could not be measured, several random non-overlapping pictures were acquired from each well using a Zeiss 710 Laser Scanning confocal microscope. Organoid perimeters for area measurements have been defined manually and by automated determination using the Analyze Particle function of ImageJ software. The sizes of the largest and smallest organoids in the reference well were measured manually, and their areas were used as the reference values for setting the minimal and maximal particle sizes. Organoids touching the edge of the images were excluded from the counting. After in culture, total organoid numbers and budding organoid numbers per well were counted by light microscopy to evaluate growth efficiency. All organoid numbers were counted manually.

\section{EdU staining}

Cell proliferation was assessed by Cell-Light EdU DNA cell proliferation kit (C103102, RiboBio), according to the manufacturer's instructions. In brief, intestinal organoids were exposed to $25 \mathrm{mM}$ of EdU for $2 \mathrm{~h}$ at $37^{\circ} \mathrm{C}$, and then the organoids were fixed in $4 \%$ paraformaldehyde. After permeabilization with $0.5 \%$ Triton $\mathrm{X}-100$, the cells were reacted with $1 \times$ Apollo reaction cocktail (RiboBio) for 30 min. Subsequently, the DNA contents of the cells were stained with DAPI for $30 \mathrm{~min}$ and visualized under a Zeiss 710 Laser Scanning confocal microscope. The numbers of $\mathrm{EdU}^{+}$cells in per organoids were analyzed by image software.

\section{Quantitative RT-PCR}

Organoids were harvested after treatment, and total RNA was extracted from the organoids using RNAiso Plus (Takara). Reverse transcription of the RNA was performed with the primers listed in Table 1. Two microliters of template RNA was reacted with TagMan PCR Master Mix for a final volume of $20 \mu \mathrm{l}$ (Takara). The thermal cycling conditions were $5 \mathrm{~min}$ at $95^{\circ} \mathrm{C}$, followed by 40 cycles of 15 $\mathrm{s}$ at $95{ }^{\circ} \mathrm{C}$ and $34 \mathrm{~s}$ at $60^{\circ} \mathrm{C}$ using an Applied Biosystems 7500 real-time PCR system.

Table 1 Primer sequences used for qRT-PCR

\begin{tabular}{llll}
\hline Target genes & Primer sense $\left(5^{\prime}-3^{\prime}\right)$ & Primer antisense $\left(5^{\prime}-3^{\prime}\right)$ & $\begin{array}{l}\text { Product } \\
\text { size }(\mathrm{bp})\end{array}$ \\
\hline mLgr5 & CCTACTCGAAGACTTACCCAGT & GCATTGGGGTGAATGATAGCA & 165 \\
mOlfm4 & CAGCCACTTTCCAATTTCACTG & GCTGGACATACTCCTTCACCTTA & 175 \\
mAscl2 & AAGCACACCTTGACTGGTACG & AAGTGGACGTTTGCACCTTCA & 115 \\
mLyz1 & GAGACCGAAGCACCGACTATG & CGGTTTTGACATTGTGTTCGC & 214 \\
mMuc2 & ACGATGCCTACACCAAGGTC & TGATCTTTACATGTTCCCA & 210 \\
mAlpi & AGGACATCGCCACTCAACTC & GGTTCCAGACTGGTACTGTCA & 128 \\
mDefa6 & CCTTCCAGGTCCAGGCTGAT & TGAGAAGTGGTCATCAGGCAC & 317 \\
mReg3g & ACTCCCTGAAGAATATACCCTCC & CGCTATTGAGCACAGATACGAG & GGC \\
mGADPH & ATGCTTCCCCGTATAACCATCA & GGCCATATCTGCATCATACCAG & 201 \\
\hline
\end{tabular}




\section{Western blot}

Different treatment organoids were lysed in RIPA buffer (50 mM Tris- $\mathrm{HCl}, \mathrm{pH} 7.4,1 \% \mathrm{NP}-40,150 \mathrm{mM} \mathrm{NaCl}$ ) containing a protease inhibitor cocktail (Thermo Fisher Scientific). Protein concentrations were detected using a BCA protein quantification kit (Thermo Fisher Scientific). Equal amounts of protein were separated by SDS-PAGE and electrophoretically transferred onto PVDF membranes (Millipore, China). After blocking with 5\% nonfat milk in TBS containing $0.1 \%$ Tween-20, the membrane was probed with rabbit anti-Lgr5 (1:1000, ab75732, Abcam) antiSTAT3 $(1: 1,000,9139$, Cell Signaling) and anti-pSTAT3 $(1: 1,000,9145$, Cell Signaling) and mouse anti-GAPDH $(1: 1,000$, SAB4300645, Sigma) for normalization. After washing, the membranes were incubated with goat antirabbit secondary antibodies (1:5000, ab203-01, Vazyme) and mouse anti-rabbit secondary antibodies (1:5000, ab20102, Vazyme). Signals were detected using a SuperSignal West Pico kit (Thermo Fisher Scientific) and subjected to an Image Reader LAS-4000 imaging system (FUJIFLIM, Japan).

\section{Cytokines detection}

The organoids were treated, and then the culture medium was obtained and stored at $-20^{\circ} \mathrm{C}$ until use for cytokine analysis. Small intestine and colon were collected from euthanized mice, and organs were then homogenized and spun down. The supernatant was stored at $-20{ }^{\circ} \mathrm{C}$ until use for cytokine analysis. Murine IL-22 (BMS6022, eBioscience), IL-1 $\beta$ (EK0394, Boster) and TNF- $\alpha$ (EK0527, Boster) were measured using an ELISA kit according to the manufacturer's instructions.

\section{Mouse experiments}

Four-week-old mice were orally administrated with PBS $(200 \mu \mathrm{L})$ or L. reuteri D8 $\left(10^{8} \mathrm{CFU}\right)$ suspended in $200 \mu \mathrm{L}$ PBS once a day, for a period of 27 days. In 21 days, colitis was induced by administering 5\% (w/v) DSS (MP Biomedicals) in drinking water for continuous 7 days, followed by normal water for two days. In 29 days, mice were sacrificed and subsequent experiments were performed. The detailed methods were listed in Fig. 3b. The body weights of the mice were recorded. The mice were sacrificed after different treatments, and then their colons were removed and the colon length was measured. Jejunal and colonic tissues were fixed with $4 \%$ paraformaldehyde, embedded in paraffin wax, sliced, and stained with hematoxylin and eosin (HE). The villus height and crypt depth of the jejunum was then measured by image $\mathbf{J}$ software. Histological pathology was detected under light microscopy. The concentrations of IL-
22 (BMS6022, eBioscience), IL-1 $\beta$ (EK0394, Boster) and TNF- $\alpha$ (EK0527, Boster) were measured using an ELISA kit. The mRNA expression of Reg3b and Reg3g in the intestine were detected by quantitative RT-PCR.

In order to verify the colonization of $L$. reuteri $\mathrm{D} 8$ in the intestine, mice were orally administrated with PBS $(200 \mu \mathrm{L})$ or $10^{8}$ CFU L. reuteri D8 suspended in $200 \mu \mathrm{L}$ PBS only once. Freshly mice feces at indicated time points $(0,4,8$, $16,24,48,72,96$, and $168 \mathrm{~h}$ ) were collected and added with $2 \mathrm{ml} \mathrm{PBS}$, and then were cultured on MRS plates containing $500 \mu \mathrm{g} / \mathrm{ml}$ tetracycline at $37^{\circ} \mathrm{C}$ for $16 \mathrm{~h}$ to count the bacterial colony. Moreover, D8 was stained with Bac Light ${ }^{\mathrm{TM}}$ Bacterial Green Stains (B-35000, Molecular Probes), according to manufacturer's instructions. In brief, $10^{8} \mathrm{CFU}$ L. reuteri D8 and $100 \mu \mathrm{M}$ working solution of the BacLight bacterial stain dissolved in DMSO were incubated for 15 min at room temperature. The D8 samples were washed with PBS to remove excess dye. Mice were administrated with PBS $(200 \mu \mathrm{L})$ or $10^{8} \mathrm{CFU}$ L. reuteri D8 labeled by Bac Light $^{\mathrm{TM}}$ Bacterial Green Stains suspended in $200 \mu \mathrm{L}$ PBS only once to detect the bacterial colonization. After 16 $\mathrm{h}$, a 2-cm section of jejunum and colon were collected from mouse, frozen immediately with liquid nitrogen, OCT embedded, and sectioned at $8 \mu \mathrm{m}$ and rinsed in HBSS, then the samples were examined with a Zeiss 710 Laser Scanning confocal microscope.

\section{Immunofluorescence assay}

A 2-cm section of jejunum and colon were collected from each mouse in different groups, fixed overnight in $4 \%$ paraformaldehyde, optimal cutting temperature compound (OCT compound) embedded, and sectioned at $8 \mu \mathrm{m}$ and rinsed in HBSS, then permeabilized with $0.5 \%$ Triton $\mathrm{X}$ 100 for $20 \mathrm{~min}$, followed by washing three times with HBSS and incubating for $1 \mathrm{~h}$ in 3\% BSA in HBSS to reduce nonspecific background. For PCNA staining, jejunum and colon sections were permeabilized and incubated with antimouse PCNA antibody (1:50, ab29, Abcam) overnight. For Paneth cell and ISCs staining, organoids and tissue sections were permeabilized and incubated with anti-mouse Lgr5 antibody (1:50, ab75732, Abcam) or anti-mouse lysozyme antibody (1:50, ab36362, Abcam) overnight. For pSTAT3 staining, organoid cells were permeabilized and incubated with anti-mouse pSTAT3 antibody $(1: 1,000$, 9139, Cell Signaling) overnight. The samples were then incubated with goat anti-rabbit Alexa Fluor 549 (1:200, ab150080, Abcam) or goat anti-mouse Alexa Fluor 488 (1:200, ab150117, Abcam) and DAPI (1:5,000, D1306, Invitrogen) for $1 \mathrm{~h}$ at room temperature. The samples were examined with a Zeiss 710 Laser Scanning confocal microscope. Fluorescence images were collected for further qualitative and quantitative analysis. The numbers of 
$\mathrm{PCNA}^{+}, \mathrm{Lgr}^{+}$and lysozyme ${ }^{+}$cells in per crypt were counted and analyzed by image $\mathrm{J}$ software.

\section{Statistical analysis}

Results were expressed as means \pm SD. One-way ANOVA was employed to determine statistical differences among multiple groups, and $t$-test was employed to determine the same between two groups. $* P<0.05$, $* * P<0.01$, $* * * P<$ 0.001. Data combined from at least three independent experiments unless otherwise stated.

Acknowledgements This study was supported by the National Natural Science Foundation of China (31502024), Jiangsu Agriculture Science and Technology Innovation Fund (JASTIF, CX[15]1066), and a project funded by the Priority Academic Program Development of Jiangsu Higher Education Institutions (PAPD).

Author contributions QH was responsible for performing the experiments, data analysis and writing the manuscript. LY established the co-cultured system of organoids and LPLs. HL and LH were responsible for animal experiments. QY provided suggestions regarding the experimental procedures. TJR give suggestions to the experiments performance and article review. QY was responsible for the conception and design of the study, data collection, drafting the article, and final approval of the version submitted.

\section{Compilance with ethical standards}

Conflict of interest The authors declare that they have no conflict of interest.

\section{References}

1. Turner JR. Intestinal mucosal barrier function in health and disease. Nat Rev Immunol. 2009;9:799-809.

2. Tumanov AV, Koroleva EP, Guo XH, Wang YG, Kruglov A, Nedospasov $\mathrm{S}$, et al. Lymphotoxin controls the IL-22 protection pathway in gut innate lymphoid cells during mucosal pathogen challenge. Cell Host Microbe. 2011;10:44-53.

3. Bloemendaal AL, Buchs NC, George BD, Guy RJ. Intestinal stem cells and intestinal homeostasis in health and in inflammation: a review. Surgery. 2016;159:1237-48.

4. Andersson-Rolf A, Zilbauer M, Koo BK, Clevers H. Stem cells in repair of gastrointestinal epithelia. Physiology. 2017;32:278-89.

5. Matricon J, Barnich N, Ardid D. Immunopathogenesis of inflammatory bowel disease. Self Nonself. 2010;1:299-309.

6. Michielan A, D'Inca R. Intestinal permeability in inflammatory bowel disease: pathogenesis, clinical evaluation, and therapy of leaky gut. Mediat Inflamm. 2015;2015:628157.

7. Kerman DH, Deshpande AR. Gut microbiota and inflammatory bowel disease: the role of antibiotics in disease management. Postgrad Med. 2014;126:7-19.

8. Hold GL, Smith M, Grange C, Watt ER, El-Omar EM, Mukhopadhya I. Role of the gut microbiota in inflammatory bowel disease pathogenesis: what have we learnt in the past 10 years? World J Gastroenterol. 2014;20:1192-210.

9. Pastorelli L, De Salvo C, Mercado JR, Vecchi M, Pizarro TT. Central role of the gut epithelial barrier in the pathogenesis of chronic intestinal inflammation: lessons learned from animal models and human genetics. Front Immunol. 2013;4:280.
10. Nigro G, Sansonetti PJ. Microbiota and gut stem cells cross-talks: a new view of epithelial homeostasis. Curr Stem Cell Rep. 2015;1:5.

11. Nigro G, Rossi R, Commere PH, Jay P, Sansonetti PJ. The cytosolic bacterial peptidoglycan sensor Nod2 affords stem cell protection and links microbes to gut epithelial regeneration. Cell Host Microbe. 2014;15:792-8.

12. Suzuki Y, Ikeda K, Sakuma K, Kawai S, Sawaki K, Asahara T, et al. Association between yogurt consumption and intestinal microbiota in healthy young adults differs by host gender. Front Microbiol. 2017;8:847.

13. Lisko DJ, Johnston GP, Johnston CG. Effects of dietary yogurt on the healthy human gastrointestinal (GI) microbiome. Microorganisms. 2017;5:pii: E6.

14. Vieira AT, Teixeira MM, Martins FS. The role of probiotics and prebiotics in inducing gut immunity. Front Immunol. 2013;4:445.

15. O'Shea EF, Cotter PD, Stanton C, Ross RP, Hill C. Production of bioactive substances by intestinal bacteria as a basis for explaining probiotic mechanisms: Bacteriocins and conjugated linoleic acid. Int J Food Microbiol. 2012;152:189-205.

16. Wang R, Jiang L, Zhang M, Zhao L, Hao Y, Guo H, et al. The Adhesion of Lactobacillus salivarius REN to a Human Intestinal Epithelial Cell Line Requires S-layer Proteins. Sci Rep. 2017;7:44029.

17. Santarmaki V, Kourkoutas Y, Zoumpopoulou G, Mavrogonatou E, Kiourtzidis M, Chorianopoulos N, et al. Survival, intestinal mucosa adhesion, and immunomodulatory potential of Lactobacillus plantarum strains. Curr Microbiol. 2017;74:1061-7.

18. de Champs C, Maroncle N, Balestrino D, Rich C, Forestier C. Persistence of colonization of intestinal mucosa by a probiotic strain, Lactobacillus casei subsp. rhamnosus Lcr35, after oral consumption. J Clin Microbiol. 2003;41:1270-3.

19. Yu Q, Yuan L, Deng J, Yang Q. Lactobacillus protects the integrity of intestinal epithelial barrier damaged by pathogenic bacteria. Front Cell Infect Microbiol. 2015;5:26.

20. Pena JA, Rogers AB, Ge Z, Ng V, Li SY, Fox JG, et al. Probiotic Lactobacillus spp. diminish Helicobacter hepaticus-induced inflammatory bowel disease in interleukin-10-deficient mice. Infect Immun. 2005;73:912-20.

21. Llopis M, Antolin M, Carol M, Borruel N, Casellas F, Martinez C, et al. Lactobacillus casei downregulates commensals' inflammatory signals in Crohn's disease mucosa. Inflamm Bowel Dis. 2009;15:275-83.

22. Barker N, van Es JH, Kuipers J, Kujala $P$, van den Born $M$, Cozijnsen $\mathrm{M}$, et al. Identification of stem cells in small intestine and colon by marker gene Lgr5. Nature. 2007;449:1003-7.

23. Sato T, Vries RG, Snippert HJ, van de Wetering M, Barker N, Stange DE, et al. Single Lgr5 stem cells build crypt-villus structures in vitro without a mesenchymal niche. Nature. 2009;459:262-5.

24. Sato T, Stange DE, Ferrante M, Vries RG, Van Es JH, Van den Brink $\mathrm{S}$, et al. Long-term expansion of epithelial organoids from human colon, adenoma, adenocarcinoma, and Barrett's epithelium. Gastroenterology. 2011;141:1762-72.

25. Wang X, Yamamoto Y, Wilson LH, Zhang T, Howitt BE, Farrow MA, et al. Cloning and variation of ground state intestinal stem cells. Nature. 2015;522:173-8.

26. Zachos NC, Kovbasnjuk O, Foulke-Abel J, In J, Blutt SE, de Jonge HR, et al. Human enteroids/colonoids and intestinal organoids functionally recapitulate normal intestinal physiology and pathophysiology. J Biol Chem. 2016;291:3759-66.

27. Liu Z, Yadav PK, Xu X, Su J, Chen C, Tang M, et al. The increased expression of IL-23 in inflammatory bowel disease promotes intraepithelial and lamina propria lymphocyte inflammatory responses and cytotoxicity. J Leukoc Biol. 2011;89:597-606.

28. Cao AT, Yao S, Gong B, Elson CO, Cong Y. Th17 cells upregulate polymeric Ig receptor and intestinal $\mathrm{IgA}$ and contribute to intestinal homeostasis. J Immunol. 2012;189:4666-73. 
29. Lindemans CA, Calafiore M, Mertelsmann AM, O'Connor MH, Dudakov JA, Jenq RR, et al. Interleukin-22 promotes intestinalstem-cell-mediated epithelial regeneration. Nature. 2015;528:560-4.

30. Sawa S, Cherrier M, Lochner M, Satoh-Takayama N, Fehling HJ, Langa $\mathrm{F}$, et al. Lineage relationship analysis of RORgammat +innate lymphoid cells. Science. 2010;330:665-9.

31. Serafini N, Wolterink RGJK, Satoh-Takayama N, Xu W, Vosshenrich CAJ, Hendriks RW, et al. Gata3 drives development of ROR gamma $\mathrm{t}(+)$ group 3 innate lymphoid cells. J Exp Med. 2014;211:199-208.

32. Sato T, van Es JH, Snippert HJ, Stange DE, Vries RG, van den Born M, et al. Paneth cells constitute the niche for Lgr5 stem cells in intestinal crypts. Nature. 2011;469:415-8.

33. El Hage R, Hernandez-Sanabria E, Van de Wiele T. Emerging trends in "Smart Probiotics": functional consideration for the development of novel health and industrial applications. Front Microbiol. 2017;8:1889.

34. Patel R, DuPont HL. New approaches for bacteriotherapy: prebiotics, new-generation probiotics, and synbiotics. Clin Infect Dis. 2015;60:S108-21.

35. Li F, Duan K, Wang C, McClain C, Feng W. Probiotics and alcoholic liver disease: treatment and potential mechanisms. Gastroenterol Res Pract. 2016;2016:5491465.

36. Schwarzer M, Makki K, Storelli G, Machuca-Gayet I, Srutkova D, Hermanova $\mathrm{P}$, et al. Lactobacillus plantarum strain maintains growth of infant mice during chronic undernutrition. Science. 2016;351:854-7.

37. Yu QH, Yuan LX, Deng J, Yang Q. Lactobacillus protects the integrity of intestinal epithelial barrier damaged by pathogenic bacteria. Front Cell Infect Mi. 2015;5:26.

38. Zelante T, Iannitti RG, Cunha C, De Luca A, Giovannini G, Pieraccini G, et al. Tryptophan catabolites from microbiota engage aryl hydrocarbon receptor and balance mucosal reactivity via interleukin-22. Immunity. 2013;39:372-85.

39. Cervantes-Barragan L, Chai JN, Tianero MD, Di Luccia B, Ahern $\mathrm{PP}$, Merriman J, et al. Lactobacillus reuteri induces gut intraepithelial $\mathrm{CD} 4+\mathrm{CD} 8$ alphaalpha $+\mathrm{T}$ cells. Science. 2017;357:806-10.

40. Kaiko GE, Ryu SH, Koues OI, Collins PL, Solnica-Krezel L, Pearce EJ, et al. The colonic crypt protects stem cells from microbiota-derived metabolites. Cell. 2016;165:1708-20.

41. Odenwald MA, Turner JR. The intestinal epithelial barrier: a therapeutic target? Nat Rev Gastroenterol Hepatol. 2016;14:9-21.

42. Perse M, Cerar A. Dextran sodium sulphate colitis mouse model: traps and tricks. J Biomed Biotechnol. 2012;2012:718617.

43. Barker N, Huch M, Kujala P, van de Wetering M, Snippert HJ, van Es JH, et al. Lgr5(+ve) stem cells drive self-renewal in the stomach and build long-lived gastric units in vitro. Cell Stem Cell. 2010;6:25-36.

44. Clevers HC, Bevins CL. Paneth cells: maestros of the small intestinal crypts. Annu Rev Physiol. 2013;75:289-311.

45. Pickert G, Neufert C, Leppkes M, Zheng Y, Wittkopf N, Warntjen $\mathrm{M}$, et al. STAT3 links IL-22 signaling in intestinal epithelial cells to mucosal wound healing. J Exp Med. 2009;206:1465-72.

46. Rendon JL, Li X, Akhtar S, Choudhry MA. Interleukin-22 modulates gut epithelial and immune barrier functions following acute alcohol exposure and burn injury. Shock. 2013;39:11-18.

47. Hanash AM, Dudakov JA, Hua G, O'Connor MH, Young LF, Singer NV, et al. Interleukin-22 protects intestinal stem cells from immune-mediated tissue damage and regulates sensitivity to graft versus host disease. Immunity. 2012;37:339-50.

48. Wang L, Fouts DE, Starkel P, Hartmann P, Chen P, Llorente C, et al. Intestinal REG3 lectins protect against alcoholic steatohepatitis by reducing mucosa-associated microbiota and preventing bacterial translocation. Cell Host Microbe. 2016;19:227-39. 